\title{
Management of Patients With Venous Thromboembolism After the Initial Treatment Period
}

\author{
Timothy M. Fernandes, MD, MPH, Manreet Kanwar, MD, and Richard White, MD
}

Background: Venous thromboembolism (VTE) is a common medical problem seen in primary care settings. The most common long-term adverse sequelae are recurrent thromboembolism and incomplete resolution of the embolic material, which may result in pulmonary hypertension after pulmonary embolism and post-thrombotic changes in the leg after deep vein thrombosis. Although there are detailed guidelines for diagnosing and treating acute VTE, there are few focused articles that provide recommendations for primary care physicians (PCPs) about how to manage VTE patients after completion of the initial period of anticoagulation treatment.

Observations: In this article, we highlight several important clinical decisions that must be addressed after the first 3 months of anticoagulation treatment is complete, with a focus on particular management issues for PCPs.

Conclusions: The 2 most important decisions the PCP must make are to determine, first, if symptoms of acute VTE have indeed resolved, and second, if they have resolved, to assess the long-term risk of recurrent VTE versus the risk of potential bleeding and decide if anticoagulation should be stopped, or if indefinite anticoagulation treatment is indicated. Among higher-risk patients who may benefit from indefinite anticoagulation, the PCP should discuss both the risks and benefits of anticoagulation treatment, empowering the patient to actively participate in this important shared decision-making process. (J Am Board Fam Med 2021;34:409-419.)

Keywords: Anticoagulants, Deep Vein Thrombosis, Dyspnea, Evidence-Based Medicine, Primary Health Care, Primary Care Physicians, Pulmonary Embolism, Pulmonary Hypertension, Shared Decision-Making, Venous Thromboembolism

\section{Introduction}

Venous thromboembolism (VTE), which includes deep vein thrombosis (DVT) and pulmonary embolism (PE), is a common medical condition that affects over a quarter of a million patients annually in the United States. ${ }^{1}$ For patients who survive an acute $\mathrm{PE}$ or DVT, the most common long-term adverse sequelae include recurrent DVT/PE and incomplete

This article was externally peer reviewed.

Submitted 28 February 2020; revised 22 August 2020; accepted 27 August 2020.

From the Division of Pulmonary and Critical Care Medicine, University of California, San Diego, San Diego (TMF); Cardiovascular Institute at Allegheny Health Network, Pittsburgh, PA (MK); and the Anticoagulation Service, UC Davis Health, Sacramento, CA (RW).

Funding: The authors received funding from Bayer Corporation for editorial assistance only in the preparation of this manuscript.

Conflict of interest: The authors report no conflicts of interest.

Disclosures: TMF: Consulting for Bayer Pharmaceuticals.

MK: Medical Advisory Board, Speakers Bureau for Bayer.

Corresponding author: Timothy $M$. Fernandes, $M D, M P H$,

Division of Pulmonary and Critical Care Medicine, University of California, San Diego, 9300 Campus Point resolution of the embolic material, ${ }^{2,3}$ which may result in pulmonary hypertension after PE and postthrombotic changes in the leg after DVT. Primary care physicians (PCPs) are often responsible for overseeing the outpatient care of these patients. ${ }^{4-6}$ Although there are detailed guidelines for diagnosing and treating acute VTE, there are few focused articles or tools available that provide PCPs with the guidance needed to manage VTE patients when the initial period of anticoagulation treatment has been completed. ${ }^{4}$ Specifically, there are several important clinical decisions that must be addressed after the first 3 months of anticoagulation treatment is complete. In this article, we identify management issues that PCPs must address and recommend a checklist (Table 1) of the major decisions that need to be made at this important juncture of their treatment.

Drive, \#7831, San Diego, CA 92037. (E-mail: tfernandes@ ucsd.edu). 
Table 1. Checklist of Important Management Decisions That Should Be Addressed after the Initial 3 Months of Anticoagulation Treatment

Essential Issues That Must Be Addressed

$\square$ Assess if signs and symptoms of the recent VTE event have resolved.

$\square$ Decide if anticoagulation therapy should be discontinued vs continued (see Table 2).

$\square$ If continued, select the duration and the dose of the anticoagulant.

$\square$ Educate the patient.

$\square$ Address patient concerns, which likely include the risk of recurrent VTE, the risk of bleeding, indications for VTE prophylaxis in the future, and the benefits of direct oral anticoagulant (DOAC) agents vs warfarin.

$\square$ Review signs and symptoms of recurrent VTE.

$\square$ Inform the patient about the radiation risks associated with repeated chest CT scanning. Inform patients about the usefulness of D-dimer testing.

Ancillary Issues

$\square$ Consider testing for antiphospholipid antibody syndrome if the results will affect future patient management.

$\square$ Order the retrieval of an inferior vena cava filter (if the patient has a deployed retrievable filter).

$\square$ Consider whether a work-up for an underlying malignancy needs to be undertaken or completed.

$\square$ Consider re-imaging the affected venous segments (upper or proximal lower extremity compression ultrasound) if the index event was a DVT and consider obtaining a D-dimer to further prognosticate the risk of VTE recurrence.

$\square$ For complex cases, is a formal thrombosis/anticoagulation consultation needed?

CT, computed tomography; DVT, deep vein thrombosis; VTE, venous thromboembolism.

*Table is based on recommendations from the authors.

\section{Clinical Presentation and Assessment}

\section{Essential Issues for Consideration}

Essential Issue \#1. Have the signs and symptoms of acute $P E$ and $D V T$ resolved? Most patients who receive the recommended 3 -month duration of anticoagulation $^{7}$ will have complete resolution of clinical symptoms and physical findings (although radiologic findings may take longer to resolve). Successful recovery following PE should manifest as restoration of normal pulmonary hemodynamics, gas exchange, and exercise tolerance. Studies have shown that most patients who receive a PE diagnosis will have nearly complete resolution of their clot burden within 6 months of anticoagulation initiation. ${ }^{8,9}$ Nonetheless, persistent dyspnea is common, with more than half of patients with a prior $\mathrm{PE}$ reporting dyspnea approximately 3 years after diagnosis. ${ }^{10}$ Of those patients reporting chronic dyspnea, many indicated they have new or worsened dyspnea since the index PE. ${ }^{10,11}$ Patients with persistent dyspnea or exercise intolerance require further evaluation. Likewise, the clot burden in patients with DVT may also fail to resolve within 3 months, leading to the development of postthrombotic symptoms or findings. Refer to Figure 1 if there are persisting symptoms of DVT or PE at the 3 -month visit.

Persisting pulmonary symptoms. Failure of $\mathrm{PE}$ to fully lyse can lead to a range of symptoms, from mild ongoing dyspnea (due to increased dead space ventilation) to severe functional limitation as a result of chronic thromboembolic pulmonary hypertension (CTEPH). The recovery after PE exists on a spectrum. A majority of patients have pulmonary artery perfusion that returns to normal or near-normal after 3 to 6 months of adequate anticoagulation, whereas some patients have some chronic clot burden with persistent intravascular obstruction distal to the acute thrombus. Approximately 30\% to 50\% of patients have some degree of persistent perfusion abnormalities detected on a ventilation/perfusion lung scan (V/Q scan) after 3 months of treatment that may cause exercise limitation and dyspnea. ${ }^{12,13}$ In a minority of these patients with chronic clot burden, the resting pulmonary artery pressures are elevated above normal, leading to CTEPH. The estimated incidence of CTEPH is $4 \%$ of all patients diagnosed with acute PE. ${ }^{14}$ However, only $7 \%$ to $29 \%$ of all patients diagnosed with CTEPH in Europe and the United States were identified in a timely fashion, suggesting that it is underdiagnosed and hence, undertreated. ${ }^{14}$

Early diagnosis and treatment of CTEPH are essential to prevent adverse effects associated with the disease. Several factors pertaining to acute PE and underlying comorbidities have been identified that increase the risk for development of CTEPH after acute $\mathrm{PE}$, specifically, having an unprovoked $\mathrm{PE}$, hypothyroidism, a delay in PE diagnosis of $>2$ weeks, and presence of right ventricular dysfunction on computed tomography angiography (CTA) at the time of the acute $\mathrm{PE}{ }^{2}$ Patients with recurrent 
Figure 1. Recommended evaluation of Dyspnea post PE.* Abbreviations: AC, anticoagulant; CTED, chronic thromboembolic disease; CTEPH, chronic thromboembolic pulmonary hypertension; PE, pulmonary embolism; RHC = right heart catheterization; TTE, transthoracic echocardiogram; V/Q, ventilation/perfusion; VTE, venous thromboembolism. Algorithm is based on recommendations from the authors and is an original figure.

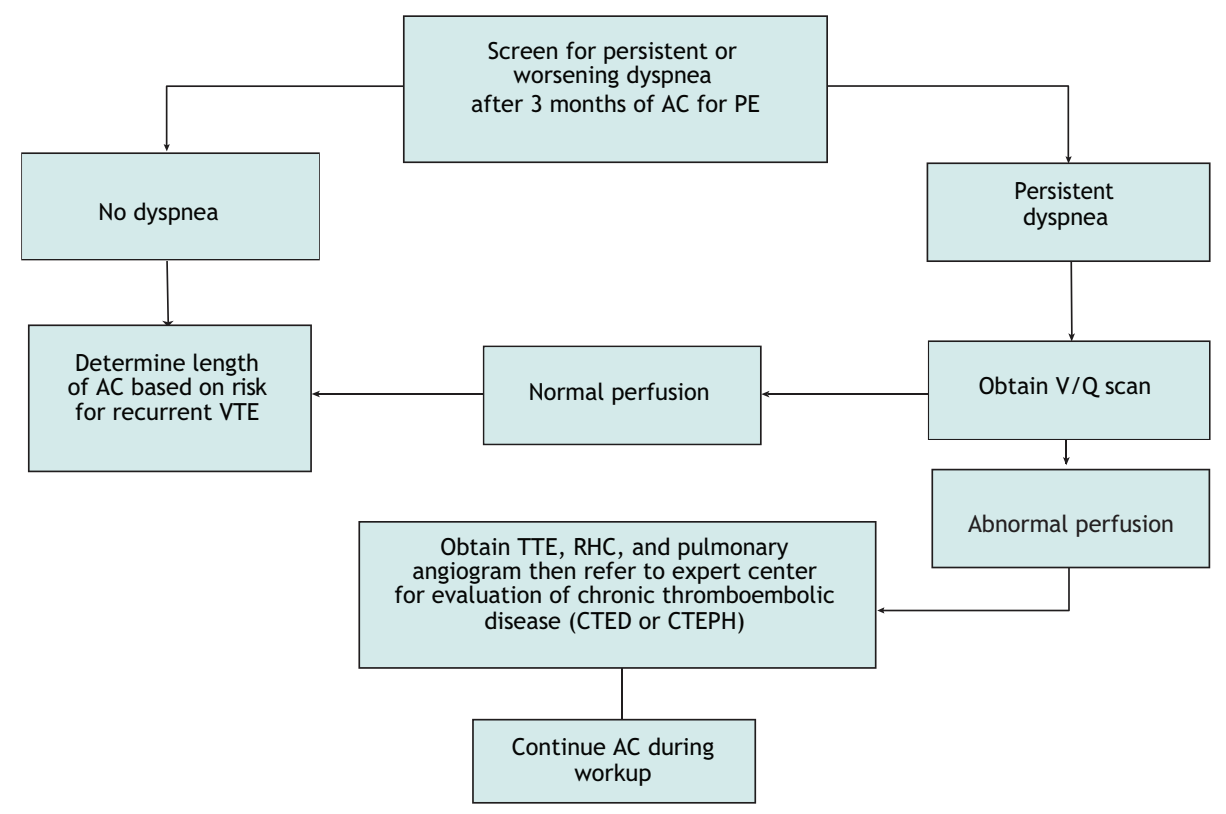

VTE also have higher odds for development of CTEPH (odds ratio: 3.2 [95\% confidence interval, 1.7 to 5.9$]) .{ }^{15}$ Patients with these risk factors merit further testing to exclude a diagnosis of CTEPH.

Importantly, in patients who do not complain of dyspnea and who say that they have returned to their pre-PE baseline status, further testing for CTEPH is not recommended after PE. ${ }^{16,17}$ This recommendation falls in line with the 2019 European Society of Cardiology/European Respiratory Society (ESC/ ERS) guidelines, which recommend only a routine clinical evaluation in PE patients 3 to 6 months after the acute PE. ${ }^{18}$ Similarly, the National Quality Forum's consensus report on VTE care quality standards recommends clinical assessment for symptoms of CTEPH but no universal screening. ${ }^{19}$ The decision to fully evaluate a patient for CTEPH should be based on the patient reporting persistent symptoms of dyspnea after 3 months of adequate anticoagulation therapy. ${ }^{17}$

Therefore, at the time of the 3-month followup visit, the PCP must specifically ask the patient if she/he has persistent dyspnea or reduced exercise tolerance. If the patient has no dyspnea, no further evaluation for CTEPH is recommended, and other criteria will be used to determine if ongoing anticoagulation therapy is necessary.
However, if a patient has persisting dyspnea, anticoagulation should be continued and further workup should be considered. ${ }^{17}$

Although some experts advocate screening symptomatic patients with transthoracic echocardiogram as the first study, ${ }^{20}$ patients with chronic thromboembolic disease may have normal resting pulmonary artery pressures reported on the echocardiogram report. ${ }^{21}$ The evaluation of possible CTEPH starts with ordering a V/Q scan as outlined in recent ESC/ERS guidelines and the 2018 World Symposium on Pulmonary Hypertension consensus. ${ }^{17,22}$ Compared with CTA, V/Q scanning has significantly better sensitivity $(51 \%$ for CTA vs $96 \%$ to $97.4 \%$ for $\mathrm{V} / \mathrm{Q}$ ) with comparable specificity ( $99 \%$ vs $90 \%$ to $95 \%$, respectively) for detecting CTEPH. Thus, V/Q is the preferred screening study-not CTA. ${ }^{23}$ Although V/Q scanning is the best test, it is underused as a follow-up test in patients with $\mathrm{PE}$. Of the $55 \%$ of patients who had a diagnostic procedural claim post-PE, only $6 \%$ had a V/Q scan compared with $47 \%$ who had a transthoracic echocardiogram. ${ }^{24}$ A normal or "low probability for PE" V/Q scan reading excludes the diagnosis of CTEPH and chronic thromboembolic disease, whereas the presence of 1 or more "high-probability" perfusion defects (more than 2 
large mismatched segmental perfusion defects) merits referral to an experienced CTEPH center for evaluation. ${ }^{25}$ At these specialized CTEPH centers, an abnormal perfusion scan is followed by pulmonary angiography and right heart catheterization to assess for pulmonary hypertension and operability. ${ }^{17} \mathrm{~A}$ formal cardiopulmonary exercise test is a sophisticated, non-invasive diagnostic test that is useful in symptomatic patients with suspected CTEPH who have normal pulmonary pressures on echocardiography. ${ }^{26}$ Among patients with dyspnea after PE, cardiopulmonary exercise testing can provide evidence of increased dead space ventilation and decreased stroke volume reserve due to persistent perfusion defects. ${ }^{27}$

Persisting leg/arm swelling or pain. Proximal DVT can lead to chronic venous obstruction or venous valvular insufficiency, which may, in turn, cause leg or arm swelling, heaviness, and edema that may not resolve within the first 3 months of anticoagulation treatment. ${ }^{28}$ This post-thrombotic syndrome may be seen in as many as $33 \%$ of patients post-DVT; the post-thrombotic syndrome is characterized by pain in the extremity involved, edema, and skin erythema and/or hyperpigmentation, with extreme cases evolving to ulcer formation in $3 \%$ of patients. ${ }^{29-31}$ If there are physical examination findings of post-thrombotic syndrome after 3 months of treatment, anticoagulation should be continued until these symptoms or findings resolve to ensure that ipsilateral recurrent DVT does not develop in that limb, which would likely worsen the problem. ${ }^{30}$

Reimaging of the deep venous system after 3 to 6 months of treatment may be useful because it serves as a better reference for future ultrasound assessment(s) of the arm or leg if symptoms persist or worsen, particularly if anticoagulation treatment is discontinued. (See Ancillary Issue \#4 below.) Measuring the level of D-dimer after completion of anticoagulation may also be useful, as it serves as a baseline referent if a D-dimer is subsequently measured because of new or worsening leg pain or swelling. ${ }^{31,32}$ In addition to continuing anticoagulation treatment, conservative measures, such as exercise training and the use of fitted gradient compression stockings, are modalities that may be useful in some patients to improve leg symptoms. $^{33,34}$ Unfortunately, a large, randomized trial (the "SOX Trial") showed that fitted gradient stockings do not prevent the development of post-thrombotic syndrome. ${ }^{28,35}$

\section{Treatment Considerations}

Essential Issue \#2. Can Anticoagulation Therapy Be Discontinued? If Not, Address Appropriate Anticoagulant Dosing and Duration of Therapy.

Determining the appropriate duration of anticoagulation therapy for VTE is an extremely important yet challenging issue for many PCPs that frequently prompts subspecialty consultation. The decisionmaking process is complex because it depends on the presence or absence of a number of risk factors, including the patient's sex, race/ethnicity, the type of VTE event (PE vs DVT), and whether the index VTE event was unprovoked or provoked by either persisting or transient major/minor risk factors. ${ }^{7,36}$ If transient or persisting, major, provoking risk factors are not present, the VTE event is classified as unprovoked.

Patients with an unprovoked VTE event have a significantly higher incidence of subsequent recurrent VTE after anticoagulation treatment is stopped compared with patients with a provoked VTE event, ${ }^{7}$ irrespective of whether the initial duration of treatment is 3 months, 6 months, 1 year, or 2 years. Recommendations for anticoagulation management after the initial 3 months of treatment for the different types of VTE events (based on current definitions from the Scientific and Standardization Committee of the International Society on Thrombosis and Hemostasis of provoked vs unprovoked VTE and the European Society of Cardiology) are shown in Figure 2. ${ }^{36,37}$ Table 2 outlines the current American College of Chest Physicians guidelines for the duration of anticoagulation therapy. ${ }^{7}$

In studies of patients with a first-time unprovoked VTE event, the cumulative incidence of recurrent VTE in men is approximately $10 \%$ in the first year and $30 \%$ to $40 \%$ in 5 years after stopping anticoagulant therapy. ${ }^{38,39}$ The probability of a recurrent VTE event is $50 \%$ higher in patients with a history of 2 unprovoked VTEs. ${ }^{40}$ Men have a 1.6-fold higher probability of developing recurrent VTE compared with women. ${ }^{41}$ Simultaneously, the patient's risk of bleeding must be assessed. According to the 2016 American College of Chest Physicians EvidenceBased Clinical Practice Guidelines, patients with 2 or more risk factors for bleeding have an annual rate of bleeding during anticoagulant treatment of over 
Figure 2. Decision tree for anticoagulation management after initial 3 months of treatment.* Abbreviations: AC, anticoagulant; APS, antiphospholipid antibody syndrome; DVT, deep vein thrombosis; Pdvt, proximal DVT; PE, pulmonary embolism; VTE, venous thromboembolism. Major transient risk factors (within 3 months before the VTE diagnosis) include surgery with general anesthesia for $>30$ minutes, bed immobilization in-hospital for 3 or more days, and cesarean section. Minor transient risk factors occurring within 2 months of VTE diagnosis include surgery with general anesthesia $<20$ minutes, hospitalization for $<3$ days with an acute illness, estrogen therapy, pregnancy/puerperium, bed rest out of the hospital for 3 or more days with an acute illness, and leg injury with reduced mobility for at least 3 days. Persisting risk factors include A) active cancer that has i) not been treated, ii) is recurrent or progressing, or iii) is actively being treated, or B) active inflammatory bowel disease. ${ }^{35}$ *Flow chart is based on recommendations from the authors and is an original figure.

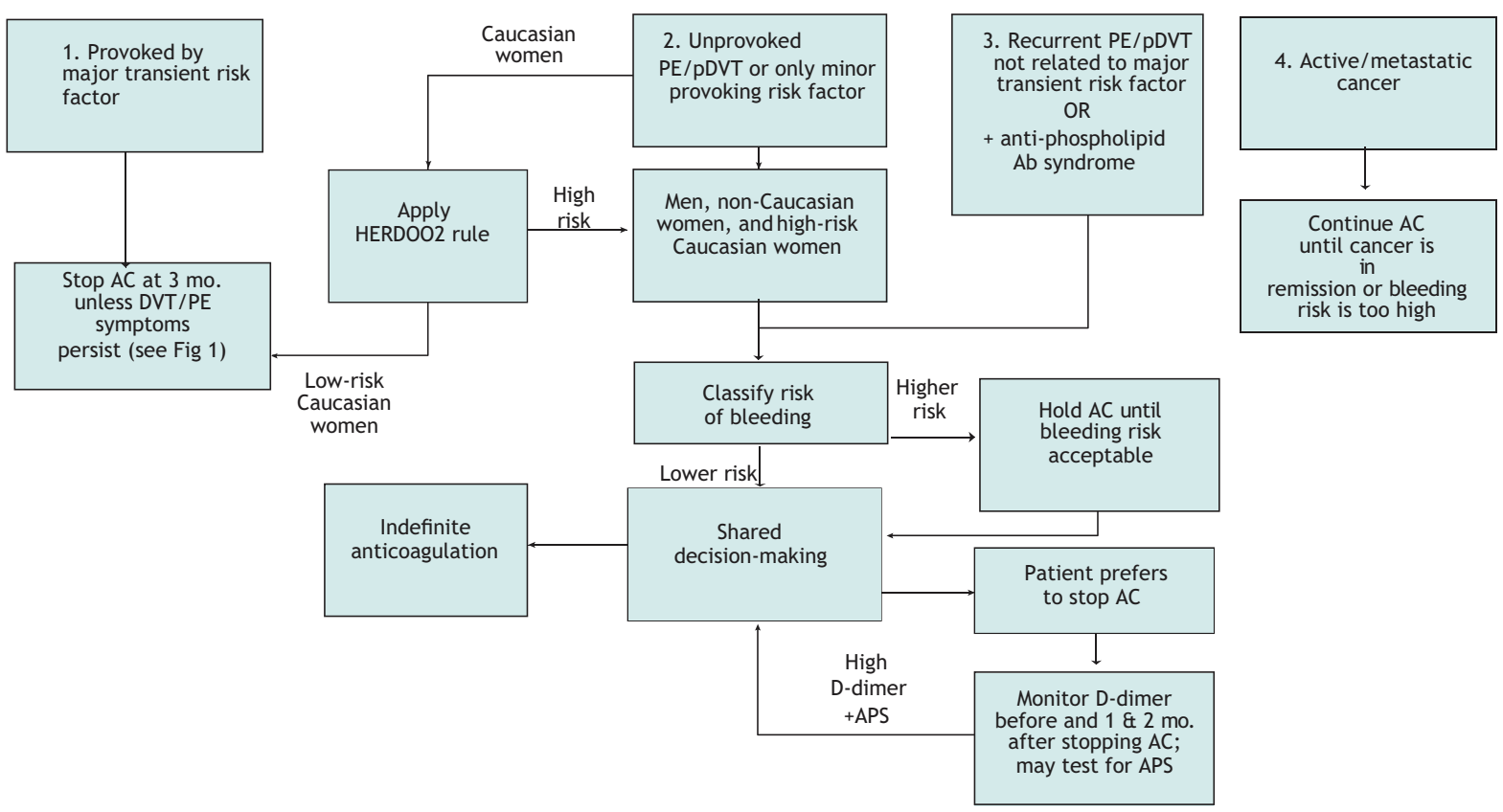

Table 2. Current Recommendations Regarding the Duration of Anticoagulation Therapy for DVT and PE

Patients treated for isolated calf-vein thrombosis

Patients with a provoked proximal DVT or PE (transient risk factor was present)

Patients with an unprovoked VTE who are at high risk for bleeding

Caucasian women with an unprovoked VTE who have 1 or fewer points using the HERDOO2 decision rule \#5
Patients with first-time proximal DVT or PE in the setting of a persisting risk factor (eg, active cancer)

Men who have a first-time unprovoked proximal DVT or PE

Caucasian women with first-time unprovoked VTE and 2 or more points using the HERDOO2 decision rule \#5

Patients who meet the criteria for having antiphospholipid antibody syndrome

Patients being evaluated for CTEPH because of ongoing dyspnea.

Until further research has been completed, the use of the HERDOO2 decision rule to risk-stratify women with unprovoked VTE should be applied only to Caucasian women.

CTEPH, chronic thromboembolic pulmonary hypertension; DVT, deep vein thrombosis; PE, pulmonary embolism; VTE, venous thromboembolism.

*Information adapted from the following sources: Rodger MA, Kahn SR, Wells PS, et al. Identifying unprovoked thromboembolism patients at low risk for recurrence who can discontinue anticoagulant therapy. Can Med Assoc 7. 2008;179(5):417-426; Konstantinides S, Meyer G, Becattini C, et al. 2019 ESC guidelines for the diagnosis and management of acute pulmonary embolism developed in collaboration with the European Respiratory Society (ERS). Eur Heart 7. 2019. doi:10.1093/eurheartj/ehz405. 
$6.5 \%$, whereas patients with just 1 risk factor have an annual rate of bleeding of $1.6 \% .^{7}$ Based on the expert opinion of the authors, if a patient has a net positive benefit, anticoagulation should be continued indefinitely, whereas if the risk of acute recurrent VTE is $<10 \%$ in 5 years, the benefit does not outweigh the risk of bleeding.

Comprehensive multivariate analysis of predictors of recurrent VTE has shown that white women with unprovoked VTE can be stratified into a low-risk group (for recurrent VTE) or high-risk group, based, respectively, on the presence of 0 to 1 or 2 or more specific risk factors. ${ }^{42}$ This 4 -component decision rule is called HERDOO2 (Hyperpigmentation, Edema, or Redness in either leg; Vidas D-dimer level $\geq 250 \mu \mathrm{g} / \mathrm{L}$; Obesity with body mass index $\geq 30 \mathrm{~kg} /$ $\mathrm{m}^{2}$; or Older age, $\geq 65$ years; 2 points for higher risk), which has been prospectively validated and can be applied only to white women who have a history of an unprovoked VTE. ${ }^{42,43}$ Those with a HERDOO2 score of $\leq 1$ have a low risk of recurrent VTE and do not need indefinite anticoagulation, whereas those with a score of $\geq 2$ have a significantly higher risk of recurrent VTE and benefit if treated with indefinite anticoagulation therapy. ${ }^{39}$ Epidemiologic studies have shown that race/ethnicity has a significant effect on the occurrence of both incident and recurrent VTE. In these studies, the only non-white group that had a significantly lower incidence of recurrent VTE that was equal to that seen in white women was African American women with unprovoked DVT (but not PE). Hispanic men and women, and African American women with PE, had a similar incidence of recurrent VTE to white men. ${ }^{44}$ Because the incidence of first-time unprovoked VTE is very low among the Asian/Pacific Islander population, insufficient data are available to analyze the incidence of recurrent VTE in this racial subgroup. ${ }^{45}$

Dosing of apixaban and rivaroxaban in VTE patients who are prescribed indefinite anticoagulation. Recent studies have compared the following strategies among patients who completed 6 to 12 months of anticoagulation treatment with a direct-acting antiXa drug for provoked or unprovoked VTE: (1) continued full-dose anti-Xa treatment; (2) half-dose anti-Xa treatment; and (3) discontinuation of anti$\mathrm{Xa}$ treatment, or (4) use of $100 \mathrm{mg}$ aspirin. ${ }^{46,47}$ Although it may be logical to think that reducing the anticoagulant dose by $50 \%$ might lead to a higher risk of recurrent DVT and a lower risk of bleeding, neither outcome was observed. There was no statistical difference in the risk of recurrent VTE for either rivaroxaban or apixaban when the full-dose treatment group was compared with the half-dose treatment group. Unfortunately, in both studies, the half-dose anti-Xa treatment group ( $2.5 \mathrm{mg}$ apixaban twice daily and $10 \mathrm{mg}$ rivaroxaban once daily) had a similar rate of major bleeding as the full-dose group, and there was no statistically significant reduction in the risk of bleeding. ${ }^{46,47}$ Continuing long-term treatment with treatment doses of dabigatran or edoxaban is another therapeutic option.

\section{Prognosis and Patient Education}

\section{Essential Issue \#3. Educate the Patient}

The 3-month visit is a good time to educate patients and address their questions and concerns. The scenario that requires the least education involves patients who have had an acute provoked VTE event following exposure to a transient risk factor, such as surgery, trauma, or immobilization/ bed rest because anticoagulation therapy can simply be discontinued in most of these patients.

For patients with an unprovoked clot or recurrent thromboembolism, current guidelines advocate shared decision making with the physician, who should first educate the patient and then discuss the risks versus the benefits of ongoing anticoagulation treatment. For patients to make informed decisions, they must have a full understanding of the risk of recurrent VTE and bleeding both on and off anticoagulation therapy.

Educational materials should describe, in layman's terms, each patient's long-term prognosis and the risks associated with either stopping anticoagulant treatment or continuing indefinite anticoagulation (Table 3). Specifically, patients should know: 1) the likelihood of developing another blood clot (eg, in 1 year, in 5 years) if they continue versus discontinuing anticoagulant therapy; 2) the magnitude of the long-term risk of bleeding associated with continued anticoagulant therapy; 3) the advantages, disadvantages, and costs of the available anticoagulants; 4) the radiation exposure associated with undergoing repeated CTA of the chest; and 5) the availability of a standing order for a D-dimer test, which can confirm (when the result is normal) no evidence indicating the formation of a blood clot.

Providing patients with a summary of the natural history of a blood clot frequently answers many of their concerns and may dispel misunderstandings. 
1. How likely they will develop another blood clot if they continue vs discontinue anticoagulation

2. The magnitude of the long-term risk of bleeding associated with anticoagulant therapy

3. The advantages, disadvantages, and costs of the available anticoagulants

4. The radiation risks associated with repeatedly undergoing CT scanning

5. The availability of a D-dimer test (when the result is normal) to confirm no new formations of a blood clot

CT, computed tomography.

*Table is based on recommendations from the authors and is an original table.

Information that is most important includes the natural history of a clot in the leg, the natural history of a clot in the lung, the risk of having a recurrent clot if anticoagulation is stopped, the risk of bleeding if anticoagulation is continued, the risk of death from a recurrent PE, and the risk of death due to bleeding. Some patients who develop a blood clot in 1 of their legs think that a blood clot always remains in their leg and that it can break off and go to the lung at any time. Other patients are concerned that any extended land or air travel will lead to the development of a new clot, even if indefinite anticoagulation is planned. These types of concerns should be addressed at the follow-up visit.

Patients who are prescribed indefinite treatment with a DOAC (eg, dabigatran, edoxaban, apixaban, rivaroxaban) may have less of an opportunity to receive 1-on-one education about the anticoagulant from a pharmacist or nurse ${ }^{48}$ compared with patients who are prescribed warfarin, who are usually formally educated by anticoagulation clinic nurses or pharmacists. There are four important points to stress about DOAC: (1) the patients must take the DOAC every day and not miss a single dose; (2) there is no clotting test (like the international normalized ratio [INR]) that indicates that they are on the correct dose of the drug; (3) it takes 2 to 3 days after stopping the DOAC for the blood level to drop to zero; and (4) they must go to an emergency department if they develop any signs or symptoms of bleeding. ${ }^{4}$ All patients should be aware of the potential bleeding risk while on anticoagulation therapy.

\section{Additional Considerations}

Ancillary Issue \#1. Consider Testing for Thrombophilic Disorders If the Results Will Affect Future Patient Management

Although patients with common thrombophilic disorders-like factor $\mathrm{V}$ Leiden or the prothrombin gene mutation-have a 2 to 3 times higher lifetime risk of having a VTE, the presence or absence of 1 of these thrombophilic disorders does not affect the risk of developing a recurrent VTE event in a patient with an unprovoked VTE compared with patients without a thrombophilic disorder who also are diagnosed with an unprovoked VTE. ${ }^{4-51}$ (In effect, all patients with a spontaneous unprovoked VTE should be thought of as likely having some underlying thrombophilic disorder.) It is not recommended that patients with unprovoked VTE be routinely tested for the presence of a thrombophilic disorder because it does not affect decision making, with the possible exception of testing for the presence of antiphospholipid antibodies (APS) which is appropriate in younger patients with either an unprovoked VTE or arterial thrombosis, thrombosis at an unusual site, late pregnancy loss, or any thrombosis or pregnancy morbidity in a patient with an autoimmune disease (especially SLE). ${ }^{52,53}$ Older patients and those with clear provoking reasons for VTE should not undergo testing for antiphospholipid antibodies. ${ }^{54}$

Patients who meet the clinical and laboratory criteria for APS seem to have a higher risk of developing recurrent VTE. ${ }^{49}$ Testing for the presence of APS includes testing for the presence of a lupus anticoagulant (LA). The most commonly performed test for the presence of an LA is the dilute Russell Viper Venom Time (dRVVT). Warfarin and the newer DOAC agents (ie, rivaroxaban, apixaban) interfere with the coagulation assays used for LA testing. ${ }^{55}$ Thus, testing for an LA requires the patient to be off warfarin for $\geq 2$ weeks and off a DOAC for $>5$ days.

However, a diagnosis of APS can be made without testing for the presence of an LA. Diagnosis of APS requires either 1) an elevated level of anticardiolipin antibody (>40 GPL or $>40$ MPL units) on 2 occasions at least 3 months apart, or 2) an elevation of 
either $\beta$-2-glycoprotein I IgG or IgM antibody (>99th percentiles) on 2 occasions at least 3 months apart. $^{56}$ Testing for an LA may be necessary, but only if 1 or both of the above tests are negative. Patients who report having a positive LA test at some time in the past (coupled with negative anticardiolipin Ab levels and $\beta$-2-glycoprotein I Ab levels) may need to be retested. Warfarin is the anticoagulant of choice for patients with APS, with the proviso that managing warfarin can be challenging if the baseline INR is prolonged by the presence of APS. ${ }^{57}$ It has been shown that point-of-care INR testing in patients with APS may also yield falsely elevated INR values, which may necessitate adjustment of the point-of-care INR values based on the corresponding chromogenic factor X levels. ${ }^{58,59}$

\section{Ancillary Issue \#2. Retrieval of an Inferior Vena Cava (IV.C) Filter}

The only indication for an IV.C filter is the presence of an acute lower extremity proximal DVT coupled with a contraindication to anticoagulant therapy. The use of an IV.C filter seems to provide some degree of improved survival in patients who cannot be anticoagulated, but it does not prevent $\mathrm{PE}$, and the use of a filter increases the long-term risk of developing recurrent lower extremity DVT. ${ }^{60}$ Other serious risks associated with IV.C filters have been described as well, such as device migration, embolization (detachment of device components), perforation of the IV.C, and filter fracture. ${ }^{61}$ If an IV.C filter needs to be removed, a removal request should be made to the hospital specialist or service that deploys/retrieves IV.C filters. An IV.C filter cannot be removed from some patients because of adherent clotting or for technical reasons.

\section{Ancillary Issue \#3. Is Any (Further) Work-up for Cancer Needed?}

Approximately $4 \%$ to $10 \%$ of patients with unprovoked VTE have an underlying (occult) cancer. ${ }^{62}$ The types of cancers that most commonly lead to the development of VTE are biologically aggressive Stage 3 or 4 adenocarcinomas or hematologic malignancies. Because most patients who have an underlying advanced cancer are diagnosed within 3 months of being diagnosed with a VTE, pursuing a cancer work-up 3 months after diagnosis of a VTE has been made is rarely an issue. ${ }^{63}$ The decision to perform a work-up for cancer ultimately depends on the PCP's clinical suspicion of cancer. ${ }^{62}$ Routine screening for occult malignancy with a CTA scan of the abdomen and pelvis is not warranted in asymptomatic patients with unprovoked VTE. In asymptomatic patients, the PCP should only focus on ensuring that patients are up to date on age-appropriate cancer screenings recommended for all healthy adults (ie, colonoscopy, mammography, skin cancer screening, and, potentially, prostate cancer screening). ${ }^{62}$

Ancillary Issue \#4. Consider reimaging the affected venous segments (upper or proximal lower extremity compression ultrasound) if the index event was a DVT, and consider obtaining a D-dimer to further prognosticate the risk of VTE recurrence. After the initial treatment period, the only sure way to know if any new recurrent DVT develops in the future is to have available an imaging study of the leg performed after the completion of anticoagulation treatment. The best evidence for a "new" DVT is the finding of an acute clot in a location where there was no clot in the past. Hence, the reimaging of the lower extremities documents the location and ultrasound characteristics of the clot at the end of the initial treatment period. In essence, it provides a new "baseline" for comparisons in the future.

D-dimer testing at the end of 3 to 6 months of anticoagulation may also be useful. ${ }^{60}$ As mentioned above, an elevated D-dimer while on anticoagulation treatment is a risk factor for recurrent VTE when applying the HERDOO2 risk stratification tool. Also, studies from Italy have shown that an elevated D-dimer level 1 and/or 2 months after discontinuation of anticoagulation therapy is a risk factor for developing recurrent DVT. ${ }^{64}$ Because a host of factors (eg, chronic liver disease, congestive heart failure, and renal insufficiency) may be associated with some elevation in the $\mathrm{D}$ dimer level, ${ }^{65}$ measuring D-dimer 1 and 2 months after stopping anticoagulation will provide the PCP with an estimate of the patient's "baseline" D-dimer level, making subsequent D-dimer testing much easier to interpret.

\section{Conclusion}

Table 1 summarizes the important management decisions that must be addressed after the initial 3month anticoagulation treatment of acute VTE. The 2 most important decisions the PCP must make are, first, to determine if symptoms of acute $\mathrm{PE}$ and DVT have resolved, and second, if they 
have resolved, to determine if the long-term risk of recurrent VTE is sufficiently high that indefinite anticoagulation treatment is indicated. Among the high-risk patients who may benefit from indefinite anticoagulation, the PCP should discuss the risks versus benefits of anticoagulation treatment to empower the patient to actively participate in the shared decision-making process. ${ }^{66}$

To see this article online, please go to: http://jabfm.org/content/ 34/2/409.full.

\section{References}

1. Heit JA. Venous thromboembolism: disease burden, outcomes and risk factors. J Thromb Haemost 2005;3:1611-717.

2. Klok FA, Dzikowska-Diduch O, Kostrubiec M, et al. Derivation of a clinical prediction score for chronic thromboembolic pulmonary hypertension after acute pulmonary embolism. J Thromb Haemost 2016;14:121-8.

3. Lang IM, Madani M. Update on chronic thromboembolic pulmonary hypertension. Circulation 2014;130:508-18.

4. Owens GM, Fine C, Harrington DW, et al. Improving transitions of care for patients with thromboembolic disease. Am J Manag Care 2014;20:S81S91. (suppl).

5. Jaffray J, Rajpurkar $M$, Sharathkumar A, et al. Transition of care for patients with venous thromboembolism: rationale, design and implementation of a quality intervention project conducted at American Thrombosis and Hemostasis Network (ATHN) affiliated sites. Thromb Res 2018;163: $146-52$.

6. Dobesh PP, Ahuja T, Davis GA, et al. Best practices for implementing venous thromboembolism prophylaxis across the continuum of care. Am J Manag Care 2018;24:S483-S488.

7. Kearon C, Akl EA, Ornelas J, et al. Antithrombotic therapy for VTE disease: CHEST Guideline and Expert Panel Report [published correction appears in. Chest. 2016;150(4):988]. Chest 2016;149:315-52.

8. den Exter PL, van Es J, Kroft LJ, Prometheus Follow-Up Investigators, et al. Thromboembolic resolution assessed by CT pulmonary angiography after treatment for acute pulmonary embolism. Thromb Haemost 2015;114:26-34.

9. Alonso-Martinez JL, Anniccherico-Sanchez FJ, Urbieta-Echezarreta MA, Garcia-Sanchotena JL, Herrero HG. Residual pulmonary thromboemboli after acute pulmonary embolism. Eur J Intern Med 2012;23:379-83.

10. Klok FA, Tijmensen JE, Haeck ML, van Kralingen KW, Huisman MV. Persistent dyspnea complaints at long-term follow-up after an episode of acute pulmonary embolism: results of a questionnaire. Eur J Intern Med 2008;19:625-9.

11. Kahn SR, Hirsch AM, Akaberi A, et al. Functional and exercise limitations after a first episode of pulmonary embolism: results of the ELOPE prospective cohort study. Chest 2017;151:1058-68.

12. Sanchez O, Helley D, Couchon S, et al. Perfusion defects after pulmonary embolism: risk factors and clinical significance. J Thromb Haemost 2010;8: 1248-55.

13. Pesavento R, Filippi I, Palla A, et al. Impact of residual pulmonary obstruction on the long-term outcome of patients with pulmonary embolism. Eur Respir J 2017;49:1601980.

14. Gall H, Hoeper MM, Richter MJ, Cacheris W, Hinzmann B, Mayer E. An epidemiological analysis of the burden of chronic thromboembolic pulmonary hypertension in the USA. Eur Respir Rev 2017;26:160121.

15. Ende-Verhaar YM, Cannegieter SC, Vonk Noordegraaf A, et al. Incidence of chronic thromboembolic pulmonary hypertension after acute pulmonary embolism: a contemporary view of the published literature. Eur Respir J 2017;49:1601792.

16. Konstantinides SV, Torbicki A, Agnelli G, et al. ESC guidelines on the diagnosis and management of acute pulmonary embolism: the Task Force for the Diagnosis and Management of Acute Pulmonary Embolism of the European Society of Cardiology (ESC). Endorsed by the European Respiratory Society (ERS). Eur Heart J 2014;35:3033-69, 2014.

17. Galie N, Humbert M, Vachiery JL, et al. ESC/ERS guidelines for the diagnosis and treatment of pulmonary hypertension: the Joint Task Force for the Diagnosis and Treatment of Pulmonary Hypertension of the European Society of Cardiology (ESC) and the European Respiratory Society (ERS). Endorsed by: Association for European Paediatric and Congenital Cardiology (AEPC), International Society for Heart and Lung Transplantation (ISHLT). Eur Heart J 2015;37:67-119, 2016.

18. Konstantinides SV, Meyer G, Becattini C, The Task Force for the diagnosis and management of acute pulmonary embolism of the European Society of Cardiology (ESC), et al. ESC guidelines for the diagnosis and management of acute pulmonary embolism developed in collaboration with the European Respiratory Society (ERS): the task force for the diagnosis and management of acute pulmonary embolism of the European Society of Cardiology (ESC). Eur Respir J. 2019;54: 1901647. 2019.

19. National Quality Forum. National Voluntary Consensus Standards for Prevention and Care of Venous Thromboembolism: Policy, Preferred Practices, and Initial Performance Measures. Washington, DC: National Quality Forum; 2006. Available at www.qualityforum.org/Publications/ 
2006/12/National_Voluntary_Consensus_Standards_for_ Prevention_and_Care_of_Venous_Thromboembolism_ Policy,_Preferred_Practices,_and_Initial_Performance_ Measures.aspx. Accessed June 7, 2019.

20. Coquoz N, Weilenmann D, Stolz D, et al. Multicentre observational screening survey for the detection of CTEPH following pulmonary embolism. Eur Respir J 2018;51:1702505.

21. Kim NH, Delcroix M, Jais X, et al. Chronic thromboembolic pulmonary hypertension. Eur Respir J 2019;53:1801915.

22. Kim NH, Delcroix M, Jenkins DP, et al. Chronic thromboembolic pulmonary hypertension. J Am Coll Cardiol 2013;62:D92-D99. (suppl).

23. Tunariu N, Gibbs S, Win Z, et al. Ventilationperfusion scintigraphy is more sensitive than multidetector CTPA in detecting chronic thromboembolic pulmonary disease as a treatable cause of pulmonary hypertension. J Nucl Med 2007;48: 680-4.

24. Tapson VF, Platt DM, Xia F, et al. Monitoring for pulmonary hypertension following pulmonary embolism: the INFORM Study. Am J Med 2016; 129:978-85.e2.

25. Sostman HD, Stein PD, Gottschalk A, Matta F, Hull R, Goodman L. Acute pulmonary embolism: sensitivity and specificity of ventilation-perfusion scintigraphy in PIOPED II study. Radiology 2008;246:941-6.

26. Held M, Grun M, Holl R, et al. Cardiopulmonary exercise testing to detect chronic thromboembolic pulmonary hypertension in patients with normal echocardiography. Respiration 2014;87: 379-87.

27. Fernandes TM, Alotaibi M, Strozza D, et al. Dyspnea post pulmonary embolism from $V_{D} / V_{T}$ and stroke volume deficits during exercise [in press]. Chest 2020;157:936-44.

28. Kahn SR, Galanaud JP, Vedantham S, Ginsberg JS. Guidance for the prevention and treatment of the post-thrombotic syndrome. J Thromb Thrombolysis 2016;41:144-53.

29. van Dongen CJ, Prandoni P, Frulla M, Marchiori A, Prins MH, Hutten BA. Relation between quality of anticoagulant treatment and the development of the postthrombotic syndrome. J Thromb Haemost 2005;3:939-42.

30. Kahn SR, Shrier I, Julian JA, et al. Determinants and time course of the postthrombotic syndrome after acute deep venous thrombosis. Ann Intern Med 2008;149:698-707.

31. Imberti D. D-dimer testing: advantages and limitations in emergency medicine for managing acute venous thromboembolism. Intern Emerg Med 2007;2:70-1.

32. Henke PK, Comerota AJ. An update on etiology, prevention, and therapy of postthrombotic syndrome. J Vasc Surg 2011;53:500-9.
33. Kahn SR, Shrier I, Shapiro S, et al. Six-month exercise training program to treat post-thrombotic syndrome: a randomized controlled two-centre trial. CMAJ 2011;183:37-44.

34. Cohen JM, Akl EA, Kahn SR. Pharmacologic and compression therapies for postthrombotic syndrome: a systematic review of randomized controlled trials. Chest 2012;141:308-20.

35. Kahn SR, Shapiro S, Wells PS, SOX trial investigators, et al. Compression stockings to prevent postthrombotic syndrome: a randomized placebo-controlled trial. Lancet 2014;383:880-8.

36. Kearon C, Ageno W, Cannegieter SC, Cosmi B, Geersing G-J, Kyrle PA, for the Subcommittees on Control of Anticoagulation, and Predictive and Diagnostic Variables in Thrombotic Disease. Categorization of patients as having provoked or unprovoked venous thromboembolism: guidance from the SSC of ISTH. J Thromb Haemost 2016;14:1480-3.

37. Konstantinides S, Meyer G, Becattini C, et al. ESC guidelines for the diagnosis and management of acute pulmonary embolism developed in collaboration with the European Respiratory Society (ERS. Eur Heart J 2019.

38. Prandoni P, Noventa F, Ghirarduzzi A, et al. The risk of recurrent venous thromboembolism after discontinuing anticoagulation in patients with acute proximal deep vein thrombosis or pulmonary embolism. A prospective cohort study in 1,626 patients. Haematologica 2007; 92:199-205.

39. Tritschler T, Wells PS. Extended therapy for unprovoked venous thromboembolism: when is it indicated? Blood Adv 2019;3:499.

40. Kearon C, Akl EA, Comerota AJ, et al. Antithrombotic therapy for VTE disease. Antithrombotic therapy and prevention of thrombosis, 9th ed: American College of Chest Physicians evidence-based clinical practice guidelines. Chest 2012;141:e419S-494. (suppl):eS-eS.

41. McRae S, Tran H, Schulman S, Ginsberg J, Kearon C. Effect of patient's sex on risk of recurrent venous thromboembolism: a meta-analysis. Lancet 2006;368: 371-8.

42. Rodger MA, Kahn SR, Wells PS, et al. Identifying unprovoked thromboembolism patients at low risk for recurrence who can discontinue anticoagulant therapy. Can Med Assoc J 2008;179: 417-26.

43. Rodger MA, Le Gal G, Anderson DR, REVERSE II Study Investigators, et al. Validating the HERDOO2 rule to guide treatment duration for women with unprovoked venous thrombosis: multinational prospective cohort management study. BMJ 2017;356:j1065.

44. White RH, Dager WE, Zhou H, Murin S. Racial and gender differences in the incidence of recurrent 
venous thromboembolism. Thromb Haemost 2006; 96:267-73.

45. White RH, Keenan CR. Effects of race and ethnicity on the incidence of venous thromboembolism. Thromb Res 2009;123:S11-S17. (suppl).

46. Agnelli G, Buller HR, Cohen A, et al. AMPLIFYEXT Investigators. Apixaban for extended treatment of venous thromboembolism. $\mathrm{N}$ Engl J Med 2013;368:699-708.

47. Weitz JI, Lensing AWA, Prins MH, et al. Rivaroxaban or aspirin for extended treatment of venous thromboembolism. N Engl J Med 2017; 376:1211-22.

48. Giannini J, Wong M, Dager W, MacDonald S, White RH. Direct oral anticoagulants are highrisk medications with potentially complex dosing. Available at http://psnet.ahrq.gov/web-mm/direct-oralanticoagulants-are-high-risk-medications-potentiallycomplex-dosing Published June 29, 2020. Accessed July 14, 2020.

49. Garcia D, Akl EA, Carr R, Kearon C. Antiphospholipid antibodies and the risk of recurrence after a first episode of venous thromboembolism: a systematic review. Blood 2013;122:817-24.

50. De Stefano V, Martinelli I, Mannucci PM, et al. The risk of recurrent venous thromboembolism among heterozygous carriers of the G20210A prothrombin gene mutation. Br J Haematol 2001; 113:630-5.

51. Shaheen K, Alraies MC, Alraiyes AH, Christie R. Factor V Leiden: how great is the risk of venous thromboembolism? Cleve Clin J Med 2012;79:265-72.

52. Middeldorp S. Evidence-based approach to thrombophilia testing. J Thromb Thrombolysis 2011;31:275-81.

53. Stevens SM, Woller SC, Bauer KA, et al. Guidance for the evaluation and treatment of hereditary and acquired thrombophilia. J Thromb Thrombolysis 2016;41:154-64.

54. Pengo V, Tripodi A, Reber G, Subcommittee on Lupus Anticoagulant/Antiphospholipid Antibody of the Scientific and Standardisation Committee of the International Society on Thrombosis and Haemostasis, , et al. Update of the guidelines for lupus anticoagulant detection. Subcommittee on Lupus Anticoagulant/Antiphospholipid Antibody of the Scientific and Standardisation Committee of the International Society on Thrombosis and Haemostasis. J Thromb Haemost 2009;7:1737-40.

55. Flieder T, Weiser M, Eller T, et al. Interference of DOACs in different DRVVT assays for diagnosis of lupus anticoagulants. Thromb Res 2018;165:101-6.
56. Gardiner C, Hills J, Machin SJ, Cohen H. Diagnosis of antiphospholipid syndrome in routine clinical practice. Lupus 2013;22:18-25.

57. Pengo V, Banzato A, Bison E, Zoppellaro G, Padayattil Jose S, Denas G. Efficacy and safety of rivaroxaban vs warfarin in high-risk patients with antiphospholipid syndrome: rationale and design of the Trial on Rivaroxaban in AntiPhospholipid Syndrome (TRAPS) trial. Lupus 2016;25:301-6.

58. Baumann Kreuziger LM, Datta YH, Johnson AD, Zantek ND, Shanley R, Reding MT. Monitoring anticoagulation in patients with an unreliable prothrombin time/international normalized ratio: factor II versus chromogenic factor $\mathrm{X}$ testing. Blood Coagul Fibrinolysis 2014;25:232-6.

59. Perry SL, Samsa GP, Ortel TL. Point-of-care testing of the international normalized ratio in patients with antiphospholipid antibodies. Thromb Haemost 2005;94:1196-202.

60. White RH, Brunson A, Romano PS, Li Z, Wun T. Outcomes after vena cava filter use in noncancer patients with acute venous thromboembolism: a population-based study. Circulation 2016; 133:2018-29.

61. US Food and Drug Administration. Inferior vena cava (IVC) filters: initial communication: risk of adverse events with long term use. 2010. Available at www.ecnmag.com/news/2010/08/inferior-venacava-ivc-filters-initial-communication-risk-adverseevents-long-term-use. Published August 9, 2010. Accessed June 2, 2020.

62. Husseinzadeh H, Carrier M. Occult cancer detection in patients with hemostatic disorder and venous thromboembolism. Thromb Res 2018;163: 242-5.

63. White RH, Chew HK, Zhou H, et al. Incidence of venous thromboembolism in the year before the diagnosis of cancer in 528,693 adults. Arch Intern Med 2005;165:1782-7.

64. Palareti G, Cosmi B, Legnani C, DULCIS (Ddimer and ULtrasonography in Combination Italian Study) Investigators, et al. D-dimer to guide the duration of anticoagulation in patients with venous thromboembolism: a management study. Blood 2014;124:196-203.

65. Kabrhel C, Mark Courtney D, Camargo CA, Jr, et al. Factors associated with positive D-dimer results in patients evaluated for pulmonary embolism. Acad Emerg Med 2010;17:589-97. Jun.

66. Hoffmann TC, Montori VM, Del Mar C. The connection between evidence-based medicine and shared decision making. JAMA 2014;312:1295-6. 